\title{
The Alcohol Catalytic Mechanism for Schiff Base 1,3-Proton Transfer
}

\author{
Yibo Shi ${ }^{1}$, Junde Xing ${ }^{1}$, Jie Li $^{1}$, Fang Zhu ${ }^{1}$, Xiaojun Fan ${ }^{1}$, and Yuchao Zhang ${ }^{2}$ \\ ${ }^{1}$ Taiyuan University of Technology \\ ${ }^{2}$ Nanjing University
}

May 11, 2021

\begin{abstract}
In order to explore the catalytic effect of alcohols on the 1,3-proton transfer of 1,1-diphenyl-N-(1-phenylethylidene) methylamine, the reaction potential energy surface was systematically studied at the theoretical level of $\omega$ B97-MV / def2-QZVPP // PBE0(D3BJ) / 6-31G**. The results show that the catalytic mechanism of benzyl alcohol can be divided into the acid channel and basic channel, in which the acid channel is the dominant one. In the first step, benzyl alcohol protonated the nitrogen atom of imine to form imine cation and benzyl alcohol anion, and the newly formed benzyl alcohol anion preferentially combined with the proton on $\mathrm{C} 1$; in the second step, benzyl alcohol continued to protonize the $\mathrm{C} 3$ atom, and the newly formed benzyl alcohol anion combined with the hydrogen on nitrogen, thus completing the whole proton migration process. By means of wave function analysis, it is proved that the stronger the hydrogen bond $(\mathrm{O}-\mathrm{H} \cdots \mathrm{N})$ is, the lower the free energy barrier is. When alcohols with lower pKa values are used as catalysts, the reaction barrier will be lower.
\end{abstract}

The Alcohol Catalytic Mechanism for Schiff Base 1,3-Proton Transfer

Yibo $\mathrm{Shi}^{1}$, Junde Xing ${ }^{1}{ }^{*}, \mathrm{Jie}^{1}{ }^{1}$, Fang Zhu ${ }^{2}$, Xiaojun Fan ${ }^{2}$, Yuchao Zhang ${ }^{3}$

School of Biomedical Engineering, Taiyuan University of Technology, Taiyuan, Shanxi. 030024, P. R. of China

School of Environmental Science and Engineering, Taiyuan University of Technology, Taiyuan, Shanxi. 030024, P. R. of China

State Key Lab of Analytical Chemistry for Life Science, School of Chemistry and Chemical Engineering, Nanjing University, Nanjing, Jiangsu. 210023, P. R. of China

Abstract: In order to explore the catalytic effect of alcohols on the 1,3-proton transfer of 1,1-diphenyl$\mathrm{N}$-(1-phenylethylidene) methylamine, the reaction potential energy surface was systematically studied at the theoretical level of $\omega$ B97-MV / def2-QZVPP // PBE0(D3BJ) / 6-31G**. The results show that the catalytic mechanism of benzyl alcohol can be divided into the acid channel and basic channel, in which the acid channel is the dominant one. In the first step, benzyl alcohol protonated the nitrogen atom of imine to form imine cation and benzyl alcohol anion, and the newly formed benzyl alcohol anion preferentially combined with the proton on $\mathrm{C}_{1}$; in the second step, benzyl alcohol continued to protonize the $\mathrm{C}_{3}$ atom, and the newly formed benzyl alcohol anion combined with the hydrogen on nitrogen, thus completing the whole proton migration process. By means of wave function analysis, it is proved that the stronger the hydrogen bond $(\mathrm{O}-\mathrm{H} \cdots \mathrm{N})$ is, the lower the free energy barrier is. When alcohols with lower $\mathrm{pK}_{\mathrm{a}}$ values are used as catalysts, the reaction barrier will be lower.

Key Words: imine; 1,3-proton transfer; reaction path; neutral catalyst; catalytic mechanism 


\section{1 | INTRODUCTION}

Because nitrogen elements are extremely widely distributed in living organisms, the synthesis of nitrogenous compounds is a long-standing subject. Imines are widespread in living organisms and are found to have significant antibacterial, antiviral, and anticancer activities. Its unique properties give it a place in catalytic reactions and medicinal chemistry.

Proton transfer reaction is considered to be one of the most important chemical processes in life, such as environmental monitoring ${ }^{1,2}$, transport of proteins in living organisms ${ }^{3}$, and the catalytic of enzymes ${ }^{4}$. There have been numerous previous studies on the mechanism of proton tautomerism, however, those on imine proton migration catalyzed by neutral catalysts have been poorly studied. Through 1,3-proton transfer, Schiff bases can play an important role in organic synthesis, such as the preparation of primary amines.

Due to the small proton radius, it can be in close contact with polar molecules and produce a strong polarization effect. Proton migration often requires the assistance of a catalyst that can bind to protons ${ }^{5}$. The activation energy of proton transfer reaction is usually affected by acidic conditions, basic conditions, ligands, electronic effects, and the coordination ability to guide groups.

For the 1,3-proton transfer of imine, catalysis by a base is a classic reaction. In most cases, the reaction rate of the catalytic process depends on the dehydrogenation efficiency of the catalyst. As the alkalinity increases, the barrier will gradually decrease. Alkoxides, metal hydrides, amines, etc. are all commonly used catalysts $^{6-9}$. The base can assist the imine deprotonation, and the $\pi$-electron pair of the imine anion formed will tend to transfer to the side of conjugation stability. At this time, the imine anion combines with protons again, completing the entire 1,3-proton transfer process. The effect of the base on electron conjugation of Schiff base has been proved by UV spectrum analysis ${ }^{10}$. Adding $\mathrm{KOH}$ to the Schiff base solution will make the Schiff base UV spectrum peak smaller, which proves that the alkaline solution can promote the formation of Schiff base anions.

The imine group has certain basicity and can meet with acid to form the salt. Most of the previous studies focused on the balance of intramolecular hydrogen bonds. Protons can migrate with the help of macrocycles and hydrogen bonds. For example, when imine groups are attached to aromatic rings, intramolecular hydrogen bonds can be formed with ortho hydroxyl groups, thus forming the tautomeric equilibrium of imines and ketenes ${ }^{11-16}$. It is difficult to migrate protons in the molecule, because the tension force of the ternary ring or the quaternion ring is too high, and it is easy to produce a high energy barrier ${ }^{17}$.

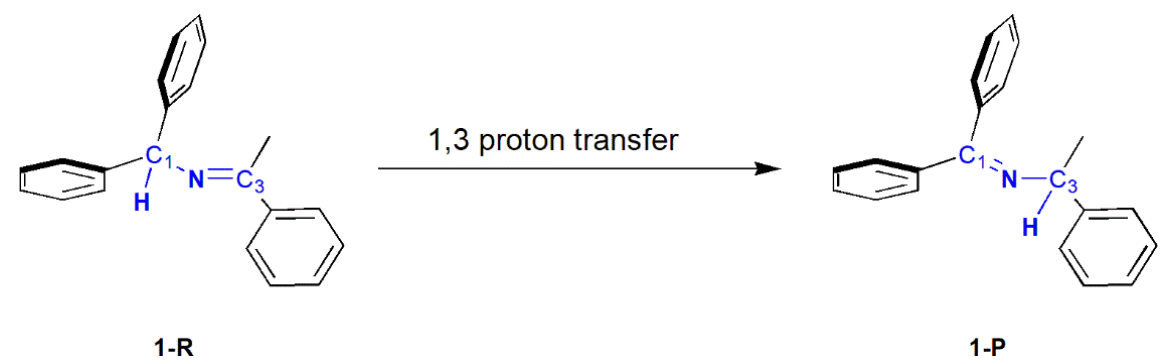

The near-neutral catalyst has the advantage of environmental friendliness. The purpose of this paper is to take the first step in the study of the proton transfer mechanism of Schiff base catalyzed by the nearneutral catalyst, to provide theoretical guidance for the experiment. Therefore, in this paper, the mechanism of proton transfer and the catalytic channel was studied in detail by using $\omega$ B97-MV / def2-QZVPP // PBE0(D3BJ) / 6-31G** method with benzyl alcohol as the catalyst. To answer the above questions, the molecular configuration, thermodynamic quantity, and energy decomposition of each stagnation point in the process of the catalytic system were studied.

Figure 1 The proton transfer reaction of 1,1-diphenyl-N-(1-phenylethylidene) methylamine 


\section{2 | COMPUTATIONAL METHODS}

All the geometric optimization, vibration analysis, transition state search and intrinsic reaction coordinate calculation in this paper were carried out by density functional theory (DFT) method with PBE0(D3BJ) / 6-31G** / cpcm / solvent ${ }^{18-22}$. All resonance frequencies were multiplied by a correction factor of $0.9779^{23}$ (sclZPE), which corrects the thermodynamic amount at $418.15 \mathrm{~K}$. Vibration frequency was computed to verify that transition state has only one negative eigenvalue. The energy of other stationary point was a local minimum on the potential energy surfaces (PESs; without any imaginary frequency). The single point energy calculation was carried out under the method of $\omega$ B97-MV / def2-QZVPP / SMD / solvent ${ }^{24-27}$, which aims to add up with the thermodynamic correction and obtain accurate energy values. The intrinsic response coordinate (IRC) analysis was performed to demonstrate the relevance of individual stagnation points. DFT calculations are executed with the RI approximation. All the above calculations were carried out under ORCA 4.2.028.

Fuzzy bond order(FBO $)^{29}$ was used to measure the number of electron pairs shared between atoms to analyze the degree of conjugation changes of covalent bonds in Schiff bases. Moreover, the hydrogen bonding evolution along the reaction pathway was analyzed based on the atoms-in-molecules (AIM) theory, which shows the topological properties of the $(3,-1)$ bond critical points (BCP) in the gradient field of the electron density. Calculation of the average local ionization energy (ALIE) was performed to predict the reaction sites $^{30}$. All wavefunction analyses were performed using Multiwfn $3.8^{31}$.

The hydrogen bonding energy was closely related to the electron density at the $\mathrm{BCP}\left(\rho_{\mathrm{BCP}}\right)^{32}$ :

$\mathrm{BE} / \mathrm{kcal} \cdot \mathrm{mol}^{-1}=-223.08 \times{ }_{B C P} / \mathrm{a} . \mathrm{u} .+0.7423(2.1)$

The 1,1-diphenyl-N-(1-phenylethylidene) methylamine discussed in this paper was all in E-configurations. The differences in optical activity were not discussed in this paper. The product N-(diphenylmethylene)-1phenylethylamine calculated were S-chirality configuration.

\section{3 | RESULTS AND DISCUSSION}




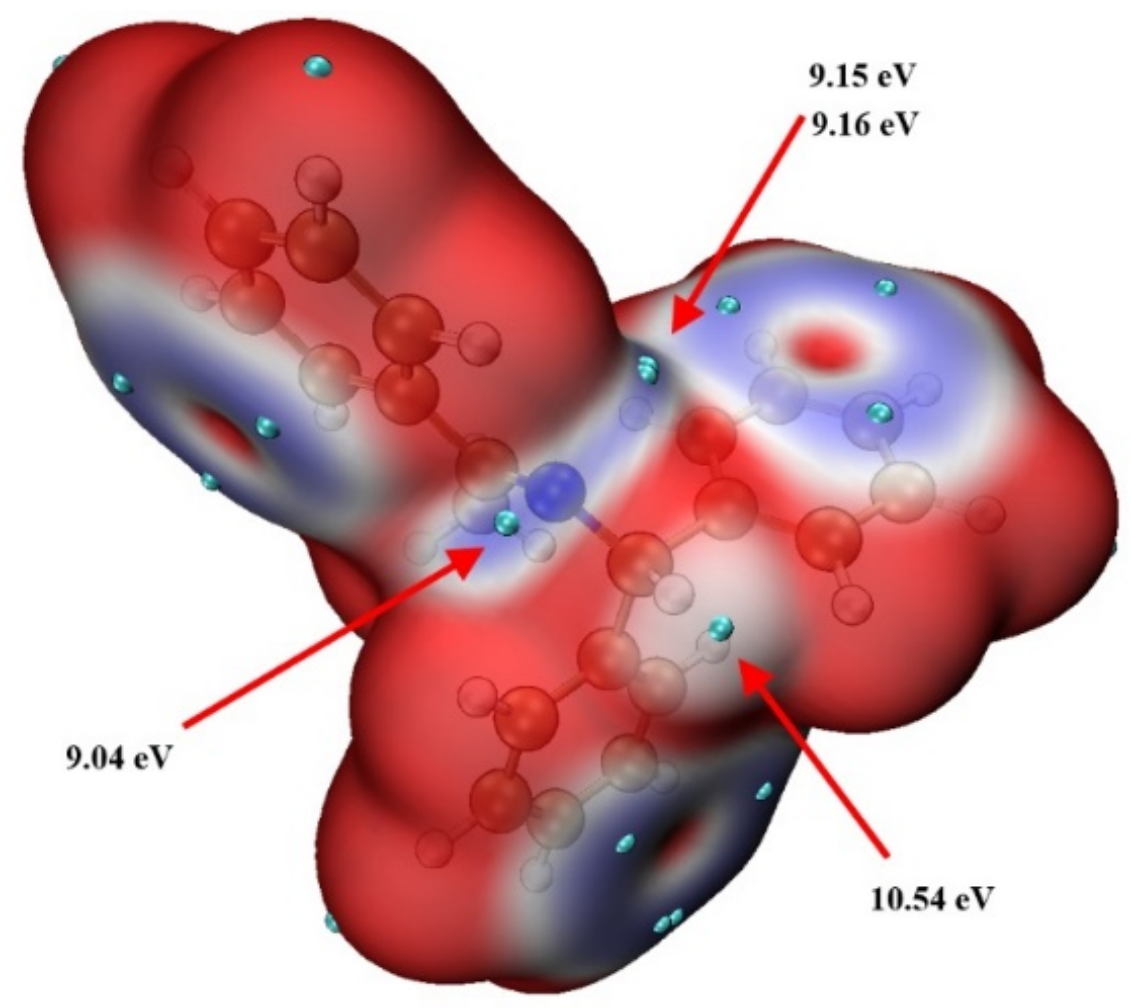

ALIE can be interpreted as the average energy required to remove electrons at a point in space, which is of great significance for investigating the activity of molecular electrophilic reaction sites. Lower ALIE values indicate that the binding force of electrons at this point is weaker, which is probably the active site. As shown in Figure 2, the global ALIE minimum value of 1,1-diphenyl-N-(1-phenylethylidene) methylamine is $9.04 \mathrm{eV}$, which appears near the nitrogen atom of the imine group. Besides the $\pi$ electron near the benzene ring, another minimum value of $10.54 \mathrm{eV}$ is near $\alpha-\mathrm{H}$ of the imine group. The results show that the imine nitrogen atom containing lone pair electrons and the hydrogen at the benzyl position is the most likely reaction site.

Figure 2. Average local ionization energy (ALIE) predicting 1,1-diphenyl-N-(1phenylethylidene) methylamine molecule (blue spheres denote the position of extremum)

\subsection{Pathways of imine proton transfer with non-catalysis}

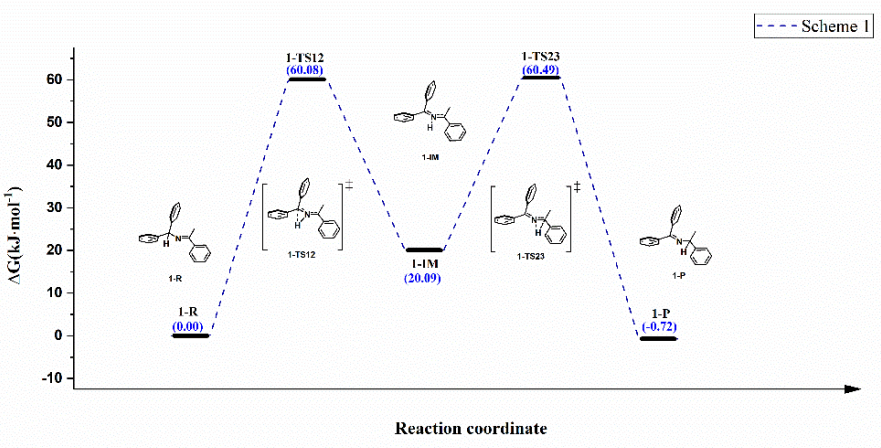


Figure 3 presents the reaction pathway for intramolecular proton migration of 1,1-diphenyl-N-(1phenylethylidene) methylamine under non-catalytic conditions. As shown in Figure 3, proton complete the 1,3-transfer process through two ternary ring transition states, starting with 1-R, going through two $\mathrm{C}-\mathrm{N}-\mathrm{H}$ constituent ternary rings to the product 1-P, in which the hydrogen atoms act with oxygen and nitrogen through electrostatic effects. The rate-determining step of the entire reaction path is from 1-R to $1-\mathrm{TS}_{2} 3^{33}$. The Gibbs free energy barrier of the process was calculated to be $60.49 \mathrm{kcal} \cdot \mathrm{mol}^{-1}$. The entire reaction is exothermic by about $0.72 \mathrm{kcal} \cdot \mathrm{mol}^{-1}$. Intramolecular proton transfer is obviously kinetically and thermodynamically unfavorable.

Figure 3 Reaction path of intramolecular proton transfer

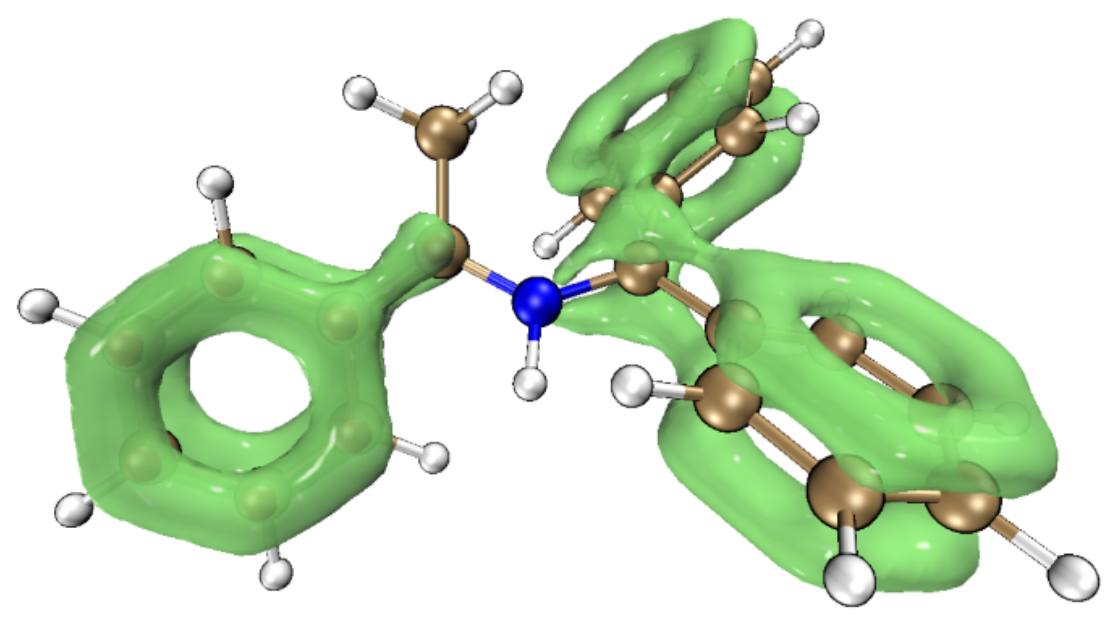

As summarized in Table 1, after proton transfer, the FBO of the C-N double bond increases (1.833-1.851), and the bond length shortens $(1.281 \mathrm{~A}-1.279 \mathrm{~A})$, which is related to the stronger electron conjugation of diphenyl. We analysis the localized orbital locator (LOL) for 1-IM. By clearing the number of $\sigma$ occupied orbits and keeping the number of $\pi$ occupied orbits unchanged, then the $\pi$ electronic characteristics are reflected $^{34}$. As shown in Figure 4, the conjugated electrons of the carbon-nitrogen bond on the biphenyl side are more than that of the monophenyl side, which indicates that the partial transfer of the carbon-nitrogen double bond has occurred at this time. For intermediate 1-IM, the carbon-nitrogen bond length (1.361A) of the diphenyl side is larger than that of the monophenyl side $(1.342 \mathrm{~A})$, which indicates that the two benzene rings have a strong potential resistance effect, which significantly stretches the bond length and hinders the electron conjugation. All of these indicate that the energy change in the process of Schiff base 1,3-proton transfer of is determined by the conjugation effect and the potential resistance effect.

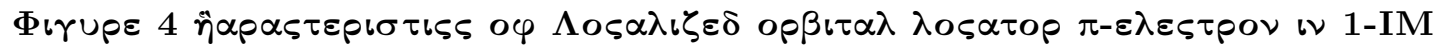

Table 1 Fuzzy bond order and key length parameters of 1-R and 1-P

\begin{tabular}{lllll}
\hline & $\mathrm{FBO}(\mathrm{C}-\mathrm{N})$ & $\mathrm{BL}(\mathrm{C}-\mathrm{N})$ & $\mathrm{FBO}(\mathrm{C}=\mathrm{N})$ & $\mathrm{BL}(\mathrm{C}=\mathrm{N})$ \\
\hline $1-\mathrm{R}$ & 1.140 & 1.459 & 1.833 & 1.281 \\
$1-\mathrm{P}$ & 1.133 & 1.465 & 1.851 & 1.279 \\
\hline
\end{tabular}

3.2 | Deprotonation assisted by benzyl alcohol reaction pathway (basic channel) 


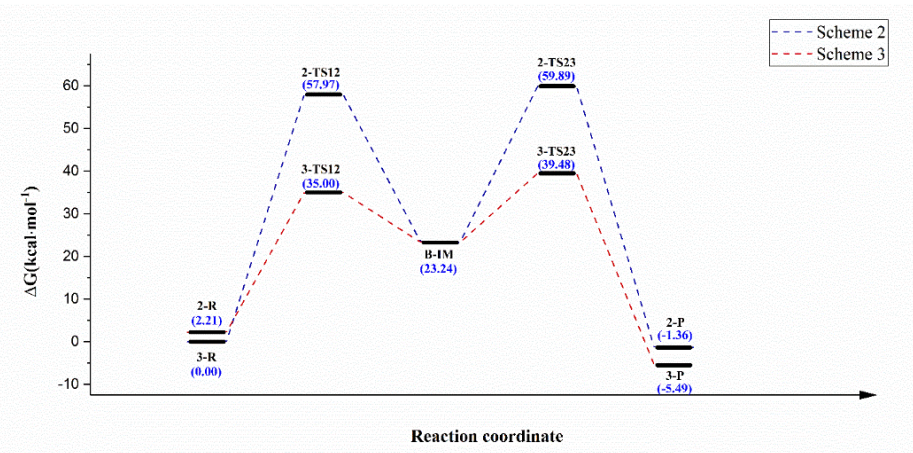

Figure 5 Gibbs free energy profile of benzyl alcohol assisted proton transfer (The blue dotted line indicates scheme 2 , and the brown dotted line indicates scheme 3 )

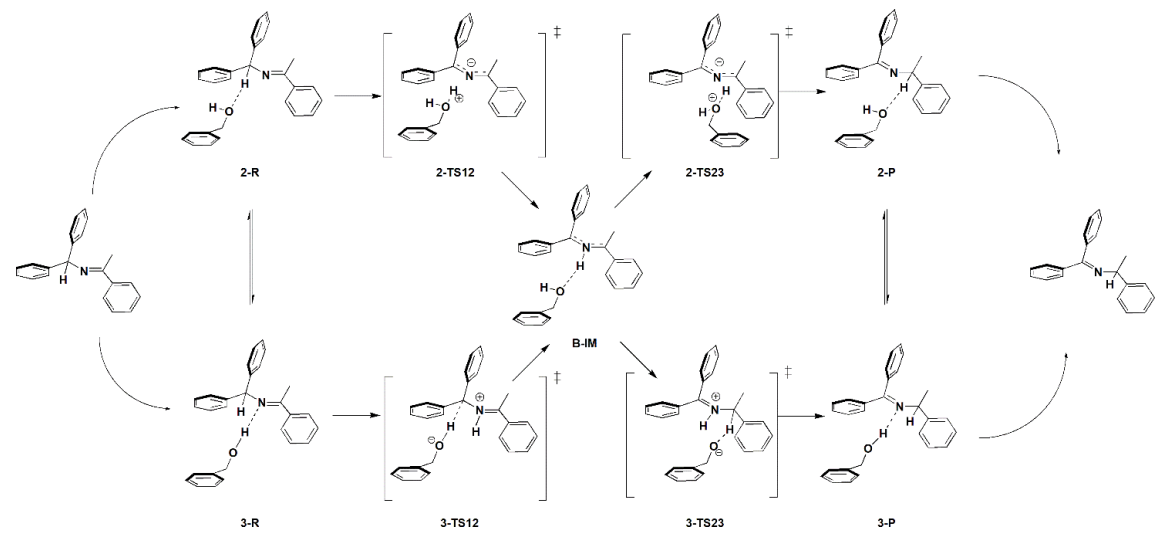

Figure 6 Reaction mechanism of benzyl alcohol as catalysis

Under the condition of non-catalytic, protons transfer through ternary rings, the ring tension is too strong that the barrier is too high. The flexible reaction system produces different complex conformations, which are conducive to the formation of different transition states. In 2-R, 1,1-diphenyl-N-(1-phenylethylidene) methylamine acts as a proton donor, and its $\alpha-\mathrm{H}$ forms a hydrogen bond with oxygen of benzyl alcohol. While in 3-R, benzyl alcohol acts as a proton donor and forms a hydrogen bond with the nitrogen atom of the imine group. The complex $3-\mathrm{R}$ is more stable than that of $2-\mathrm{R}\left(\Delta \mathrm{G}=-2.21 \mathrm{kcal} \cdot \mathrm{mol}^{-1}\right)$. This is because the electronegativity of the nitrogen atom is stronger than that of carbon and the $\mathrm{O}-\mathrm{H} \ldots \mathrm{N}$ hydrogen bond is stronger than the $\mathrm{C}-\mathrm{H}$... O. 


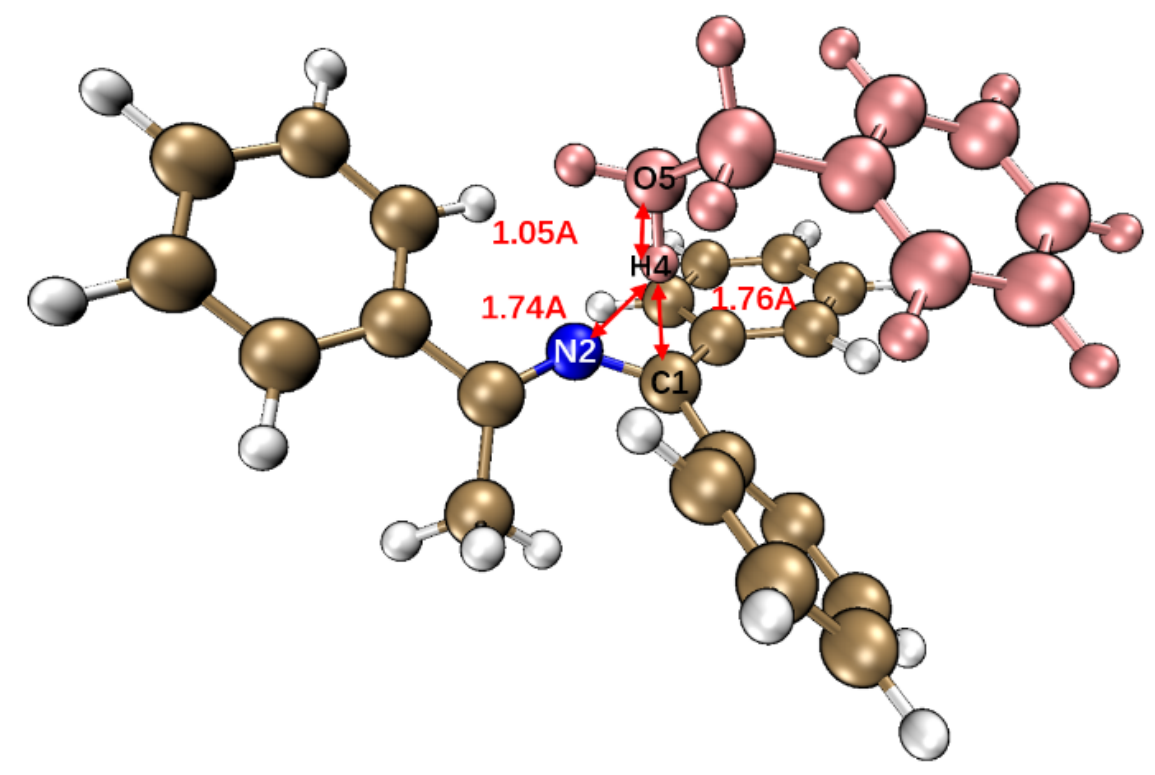

The stagnation point on potential energy surface of Scheme 2 are shown in Figure 5 and 6 . Starting from the bimolecular complex 2-R, it has been deprotonated at 2-TS12. According to the bond parameters shown in Figures 7 and 8, the bond length (BL) of $\mathrm{O}-\mathrm{H}(1.05 \mathrm{~A})$ is much smaller than that of $\mathrm{N}-\mathrm{H}(1.74 \mathrm{~A})$ and $\mathrm{C}-\mathrm{H}$ $(1.76 \mathrm{~A})$, which indicates that benzyl alcohol cation and Schiff base anion have been formed at this time. Benzyl alcohol cation attacks the nitrogen atom to form B-IM. Then benzyl alcohol continued to combine with the active protons on the nitrogen atom of Schiff base, deprotonated at 2-TS23, and transferred the protons to the carbon atom at the other end to form 2-P. The free energy change $(\Delta \mathrm{G})$ of the whole process is $-3.57 \mathrm{kcal} \cdot \mathrm{mol}^{-1}$, which indicates that this path is thermodynamically feasible. Compared with the noncatalytic, the free energy barrier $\left(57.67 \mathrm{kcal} \cdot \mathrm{mol}^{-1}\right)$ is not significantly reduced by benzyl alcohol. Obviously, this energy barrier cannot be crossed under normal experimental conditions.

Figure 7 Geometry and some bond parameters of 2-TS12 (to compare with Schiff base, benzyl alcohol molecule is all marked in pink) 


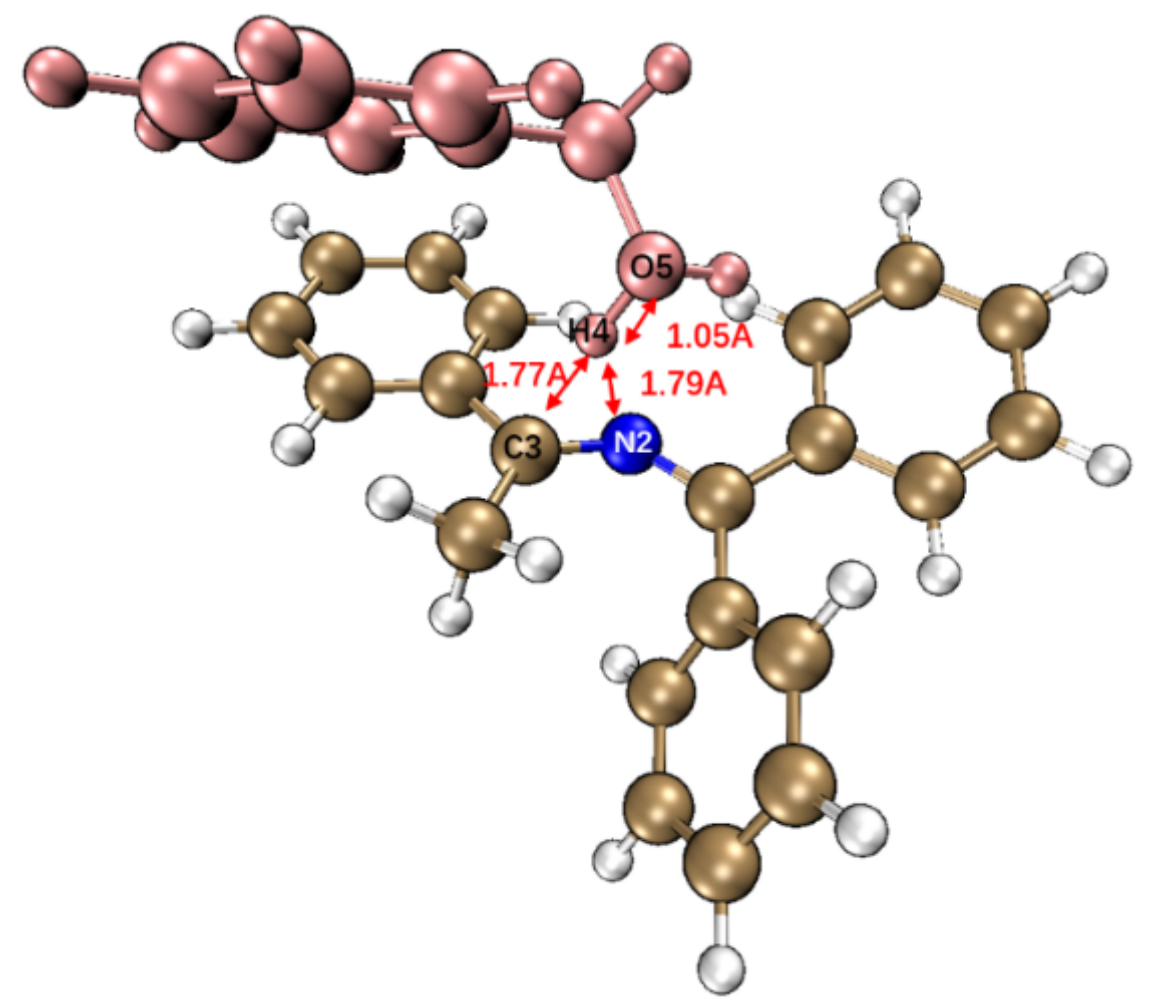

Figure 8 Geometry and bond parameters of 2-TS23

\subsection{Benzyl alcohol protonated imine reaction pathway (acid channel)}

It is shown from Table 2 that the bond angles (BA) of $\mathrm{O}-\mathrm{H} \cdots \mathrm{N}$ in $3-\mathrm{R}$ and 3-P are 175.750 and 174.360 respectively. It is generally believed that the closer the bond angle of $\mathrm{H}$-bond $(\mathrm{X}-\mathrm{H} \cdots \mathrm{Y})$ is close to $180^{\circ}$ and the more favorable for electron transmission, the easier the $\mathrm{C}-\mathrm{H}$ bond will break. The more reasonable hydrogen-bonding configuration makes proton forward migration easier than the reverse, which promotes the reaction.

In Figure 6, Scheme 3 represents the stagnation points on the acid channel. Starting from complex 3-R, benzyl alcohol is deprotonated along the direction of hydrogen bond to form benzyl alcohol anion. Benzyl alcohol acts as a base to capture the $\alpha-\mathrm{H}$ of the imine group in 3-TS12. As shown in the intrinsic reaction coordinate, the reaction pathway is divided into two stages, but there is only one maximum, which undergoes a single transition multistep reaction. Benzyl alcohol molecules capture $\alpha-\mathrm{H}$ directly after protonated nitrogen to form B-IM. Next, benzyl alcohol protonated the carbon atom on the other side at 3-TS23. Benzyl alcohol anions continue to capture hydrogen on nitrogen and complete the whole proton transfer process. 


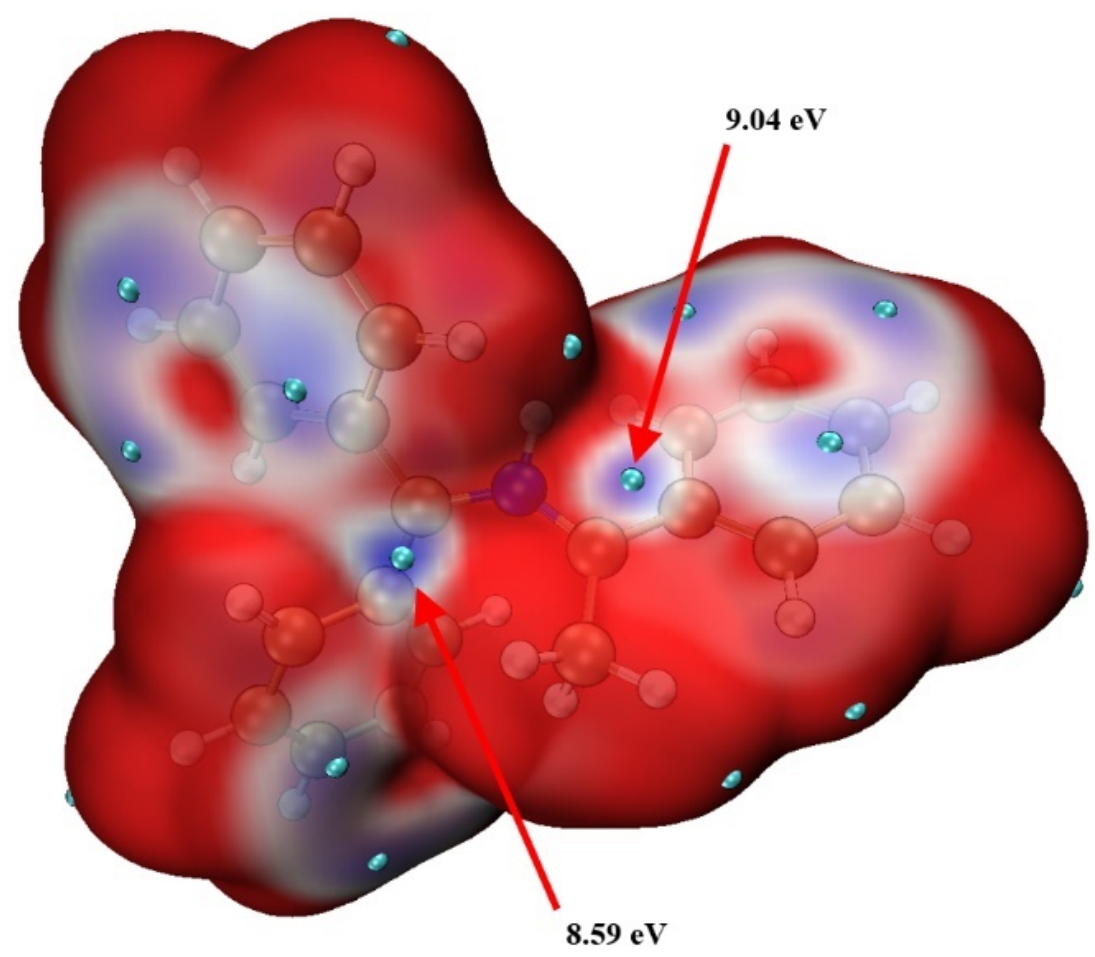

B-IM has a $\mathrm{C}_{1}-\mathrm{N}-\mathrm{C}_{3}$ delocalized $\pi$ bond with three centers and four electrons. It is not a stable structure and tends to transition to 1-R and 1-P at the same time. Figure 9 shows the ALIE value of 1-IM of Schiff base intermediate. In addition to the minimum value of ALIE distributed on the surface of the benzene ring, it is also distributed on $\mathrm{C}_{1}$ and $\mathrm{C}_{3}$, and the kinetic drive the reaction generates more 1-R, while the thermodynamically drive the reaction generates more 1-P (Figure 3). Therefore, the reaction is suitable for heating. Scheme 3 has a lower Gibbs free energy barrier $\left(39.48 \mathrm{kcal} \cdot \mathrm{mol}^{-1}\right)$, and the whole process is exergonic in free energy $\left(-5.49 \mathrm{kcal} \cdot \mathrm{mol}^{-1}\right)$. Benzyl alcohol protonated nitrogen transfer has a smaller potential barrier (Scheme 3) than deproton transfer (Scheme 2), which is a more advantageous reaction channel. It is preliminarily speculated that alcohols with lower $\mathrm{pK}_{\mathrm{a}}$ value can catalyze 1,3-proton transfer faster.

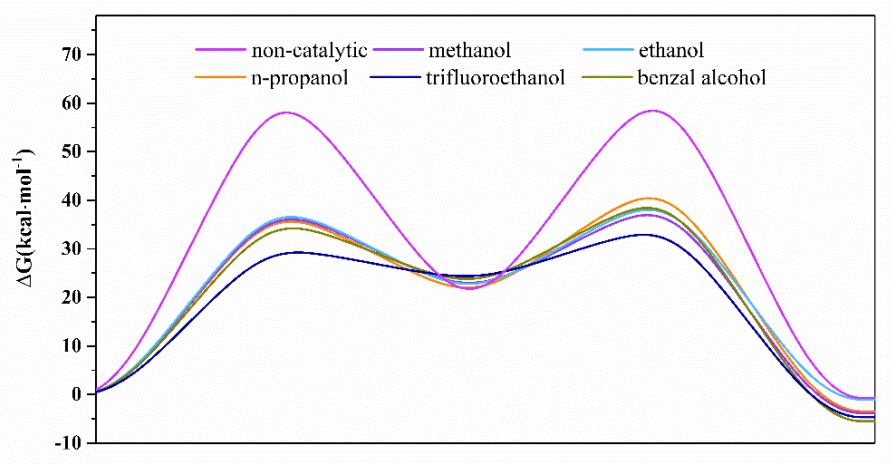

Figure 9. The plot of reaction site of 1-IM predicted by average local ionization energy (ALIE) 
Figure 10 Gibbs free energy profiles of proton transport assisted by different alcohols

\subsection{Analysis of the catalytic effect of different alcohols}

The catalytic effects of alcohols of different acidity, such as methanol $\left(\mathrm{pK}_{\mathrm{a}}=15.7\right)$, ethanol $\left(\mathrm{pK}_{\mathrm{a}}=15.9\right)$, trifluoroethanol $\left(\mathrm{pK}_{\mathrm{a}}=12.4\right)$, and $\mathrm{N}$-propanol $\left(\mathrm{pK}_{\mathrm{a}}=16.1\right)$, were also examined. Alcohols such as methanol and benzyl alcohol have different catalytic effects on 1,3-proton transfer, mainly related to the acidity of alcohols and the strength of hydrogen bond formation.

With the decrease of the $\mathrm{pK}_{\mathrm{a}}$ value, the acid strength increases, and the reaction free energy barrier decreases. As shown in Figure 10, the $\mathrm{pK}_{\mathrm{a}}$ (12.4) of trifluoroethanol is the lowest, and the reaction energy barrier is the most obvious, which is reduced from $60.08 \mathrm{kcal} \cdot \mathrm{mol}^{-1}$ to $33.61 \mathrm{kcal} \cdot \mathrm{mol}^{-1}$. It can be seen from Table 2 that the hydrogen bond energy of product $(\mathrm{P})$ in each dominant solvent is less than that of reactant $(\mathrm{R})$, which proves that the forward energy barrier of alcohol catalyzing this kind of reaction is less than the reverse energy barrier, while the hydrogen bond of trifluoromethane is the strongest $\left(-10.39 \mathrm{kcal} \cdot \mathrm{mol}^{-1}\right)$, resulting in the lowest forward and reverse energy barrier. The reason for the significant difference in the catalytic activity of different alcohols is the difference in the ability to dissociate protons. The higher the acidity of alcohol, the easier the dissociation of protons, that is, the more favorable the formation of hydrogen bond $\mathrm{X}-\mathrm{H} \cdots \mathrm{Y}$, and the smaller the energy barrier of $\mathrm{H}$ migrating to the imine nitrogen atom.

Table $2 \mathrm{O}-\mathrm{H}$... N H-bond parameters of complexes of different alcohols and 1,1-diphenyl-N-(1phenylethylidene) methylamine (The reactant and product in the methanol catalytic pathway are abbreviated as M-R and M-P. Ethanol, n-propanol and trifluoroethanol are similarly abbreviated. The BL refers to the bond length of $\mathrm{N} \cdots \mathrm{H}$, and the $\mathrm{BA}$ refers to the bond angle of $\mathrm{O}-\mathrm{H} \cdots \mathrm{N})$

\begin{tabular}{lllll}
\hline & $\rho(\mathrm{BCP}) * 10^{3}$ & $\mathrm{BE}\left(\mathrm{kcal}^{*} \mathrm{~mol}^{-1}\right)$ & $\mathrm{BL}(\mathrm{A})$ & $\mathrm{BA}$ \\
\hline $3-\mathrm{R}$ & 44.49 & -9.18 & 1.78 & 176.11 \\
3-P & 40.42 & -8.27 & 1.82 & 174.78 \\
M-R & 44.48 & -9.18 & 1.78 & 172.37 \\
M-P & 44.14 & -9.10 & 1.78 & 172.96 \\
E-R & 41.06 & -8.42 & 1.81 & 175.74 \\
E-P & 40.06 & -8.19 & 1.83 & 174.64 \\
N-R & 47.55 & -9.87 & 1.77 & 172.85 \\
N-P & 40.43 & -8.27 & 1.82 & 172.54 \\
T-R & 49.90 & -10.39 & 1.72 & 173.95 \\
T-P & 46.71 & -9.68 & 1.76 & 173.80 \\
\hline
\end{tabular}

\section{4 | CONCLUSIONS}

The alcohol catalytic pathway of 1,3-proton transfer of 1,1-diphenyl-N-(1-phenylethylidene) methylamine was studied at the $\omega$ B97-MV / def2-QZVPP // PBE0(D3BJ) / 6-31G** level. The major conclusions can be drawn as follows.

(1) When the imine group has $\alpha-\mathrm{H}$, there are two kinds of tautomerism. In this paper, the tautomers of 1,1diphenyl-N-(1-phenylethylidene) methylamine and N-(diphenylmethylene)-1-phenylethylamine are studied. It is proved that protons can overcome the steric hindrance effect and migrate to the conjugated stable side, which is consistent with the calculated value of free energy change.

(2) In the absence of catalyst, proton transfer of Schiff base has a large free energy barrier, which is considered difficult to occur. The catalytic effect of benzyl alcohol greatly reduces the barrier height, which makes the reaction easier to occur. When benzyl alcohol is used as a catalyst, the free energy barrier of Scheme 3 is lower than that of Scheme 2, which proves that the acid channel catalyzed by benzyl alcohol is better than the basic channel. 
(3) Alcohols can significantly reduce the free energy barrier, and the catalytic effect is proportional to the acid strength of alcohols. The smaller the $\mathrm{pK}_{\mathrm{a}}$ value is, the lower the reaction free energy barrier is. The reason may be that the higher the acidity of alcohol is, the more favorable it is for the fracture of $\mathrm{O}-\mathrm{H}$ and the formation of $\mathrm{N} \cdots \mathrm{H}$ in the hydrogen bond $\mathrm{O}-\mathrm{H} \cdots \mathrm{N}$, that is, the more favorable it is for the migration of $\mathrm{H}$ protons.

\section{References}

1. W. Lindinger, A. Hansel, A. Jordan, Int. J. Mass Spectrom. Ion Processes 1998 , 173, 191.

2. J. D. Gouw, C. Warneke, Mass Spectrom. Rev. 2010, 26, 223.

3. C. A. Wraight, BBA-bioenergetics $2006,1757,886$.

4. P. Brzezinski, A. L. Johansson, BBA-bioenergetics 2010 , 1797, 710.

5. M. Eigen, Angew. Chem. Int. Edit. $2010,3,1$.

6. Y. Wu, L. Hu, Z. Li, L. Deng, Nature 2015, 523, 445.

7. D. A. Jaeger, D. J. Cram, J. Am. Chem. Soc. 1971, 93, 5153.

8. A. Hjelmencrantz, U. J. Berg, J. Org. Chem. 2002, 67, 3585.

9. G. Cainelli, A. Trere, P. P. Boyl, et al., J. Org. Chem.1996 , 61, 5134.

10. L. A. Rahman, A. M. Farhan, A. R. Al-Taee, et al., Asian. J. Chem.2016 , 28, 765.

11. S. Sharif, G. S. Denisov, M. D. Toney, et al., J. Am. Chem. Soc.2006 , 128, 3375.

12. M. Rubčić, K. Užarević, I. Halasz, et al., Chem. Eur. J.2012, 18, 5620.

13. A. Mandal, D. Fitzmaurice, E. Waghorne, et al., Spectrochim. Acta A2004, 60, 805.

14. Y. L. Lin, J. Gao, Biochemistry, 2010 , 49, 84.

15. K. Aleksander, Int. J. Mol. Sci. 2003 , 4, 434.

16. M. Fores, M. Duran, M. Sola, Chem. Phys. 2000 , 260, 53.

17. S. I. Okovytyy, L. K. Sviatenko, A. A. Gaponov, et al., Eur. J. Org. Chem. 2010 , 2, 280.

18. C. Adamo, V. Barone, J. Chem. Phys. 1999, 110, 6158.

19. W. J. Hehre, R. Ditchfield, J. A. Pople, J. Chem. Phys. 1972 , 56, 2257.

20. S. Grimme, J. Antony, S. Ehrlich, et al., J. Chem. Phys.2010 , 132, 154104.

21. S. Grimme, S. Ehrlich, L. Goerigk, J. Comput. Chem. 2011, 32, 1456.

22. V. Barone, M. Cossi, J. Phys. Chem. A 1998 , 102, 1995.

23. J. P. Merrick, D. Moran, L. Radom, J. Phys. Chem. A 2007, 111, 11683.

24. N. Mardirossian, M. Head-Gordon, J. Chem. Phys. 2016 , 144, A1133.

25. F. Weigend, Phys. Chem. Chem. Phys. 2006 , 8, 1057.

26. F. Weigend, F. Furche, R. Ahlrichs, J. Chem. Phys. 2003, 119, 12753.

27. A. V. Marenich, C. J. Cramer, D. G. Truhlar, J. Phys. Chem. B2009, 113, 6378.

28. F. Neese, Wires. Comput. Mol. Sci. 2018 , 8, e1327.

29. I. Mayer, P. Salvador, Chem. Phys. Lett. $2004,383,368$.

30. S. Per, S. M. Jane, B. Tore, et al., Can. J. Chem. 1990 , 68:1440.

31. Lu, T. Chen, F. W. J. Comput. Chem. 2012, 33, 580.

32. S. Emamian, T. Lu, H. Kruse, et al., J. Comput. Chem. 2019 , 40, 2868.

33. J. R. Murdoch, J. Chem. Educ. 1981, 58, 32.

34. T. Lu, Q. X. Chen, Theor. Chem. Acc. 2020, 139, 25.

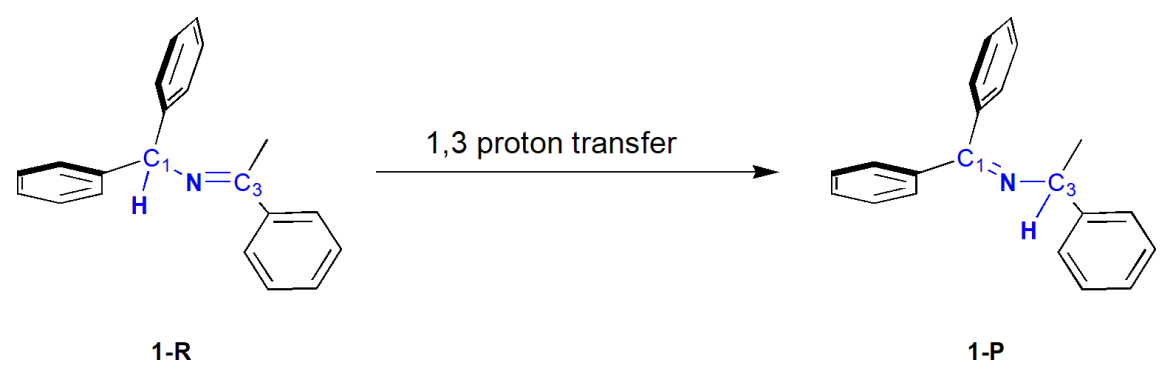



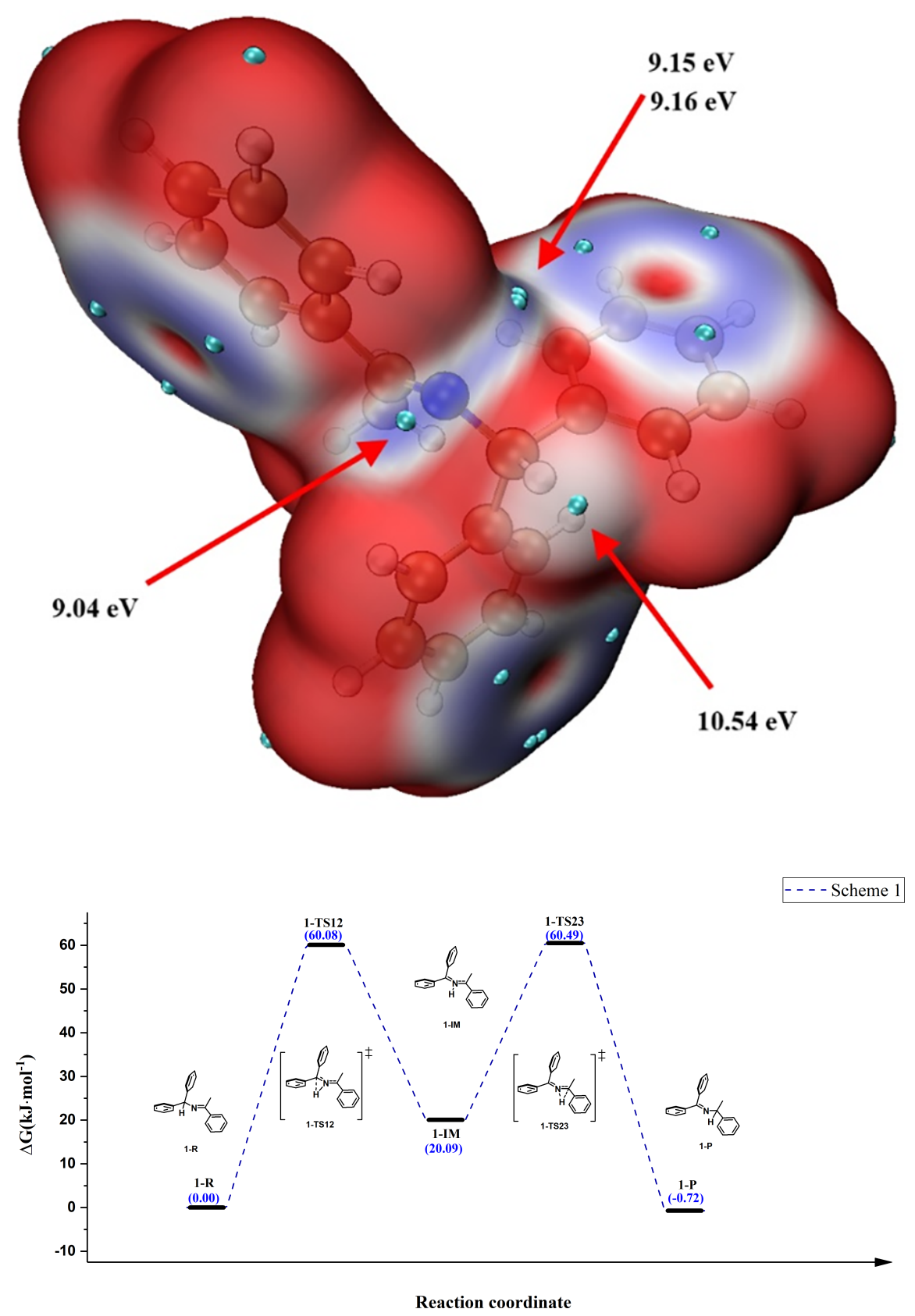

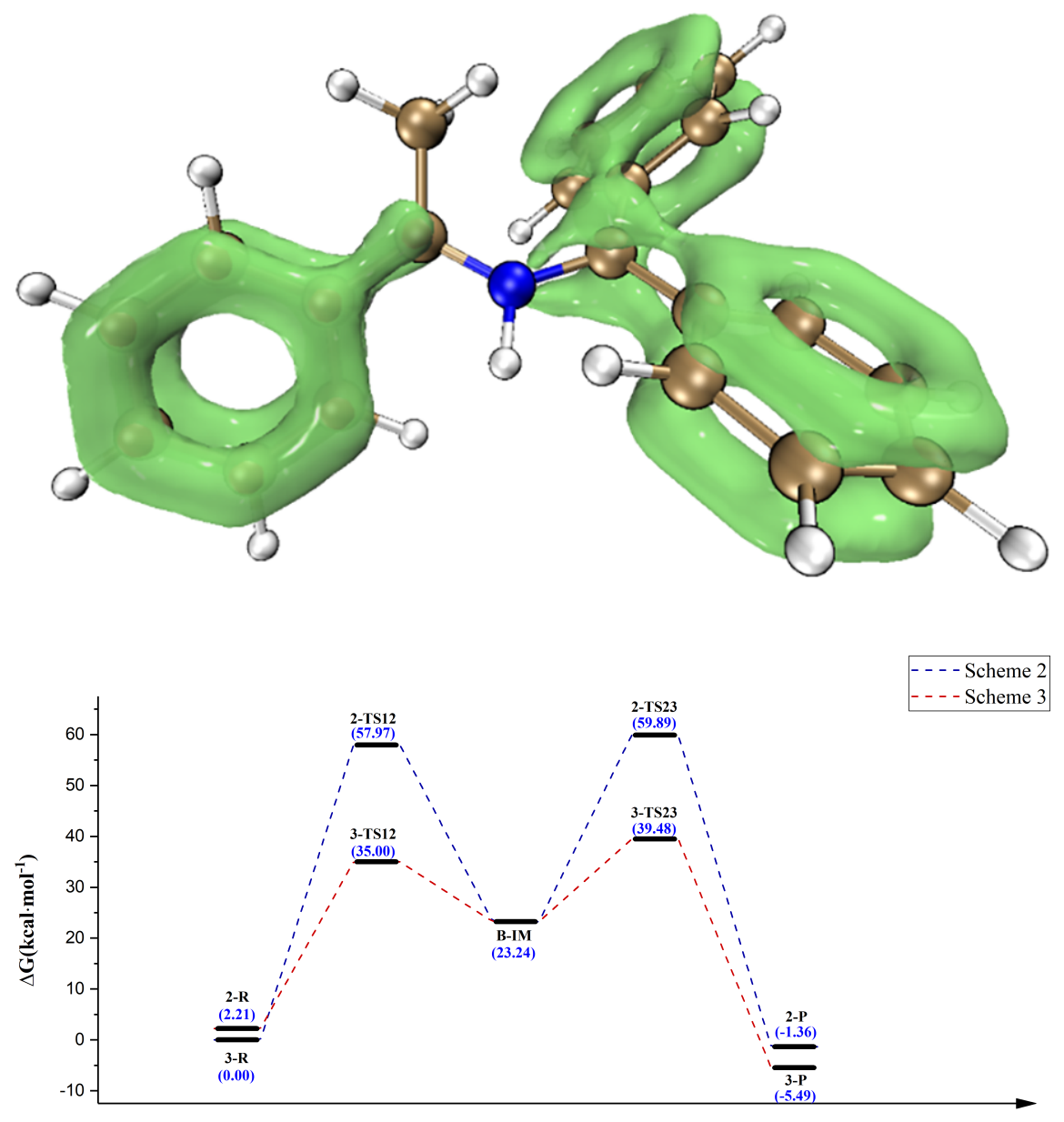

Reaction coordinate

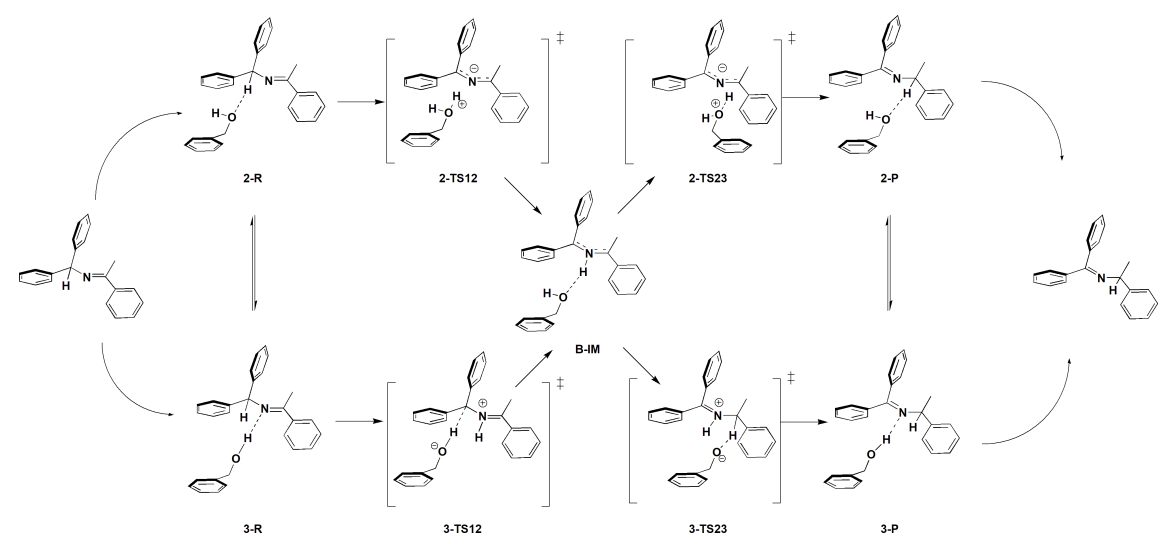



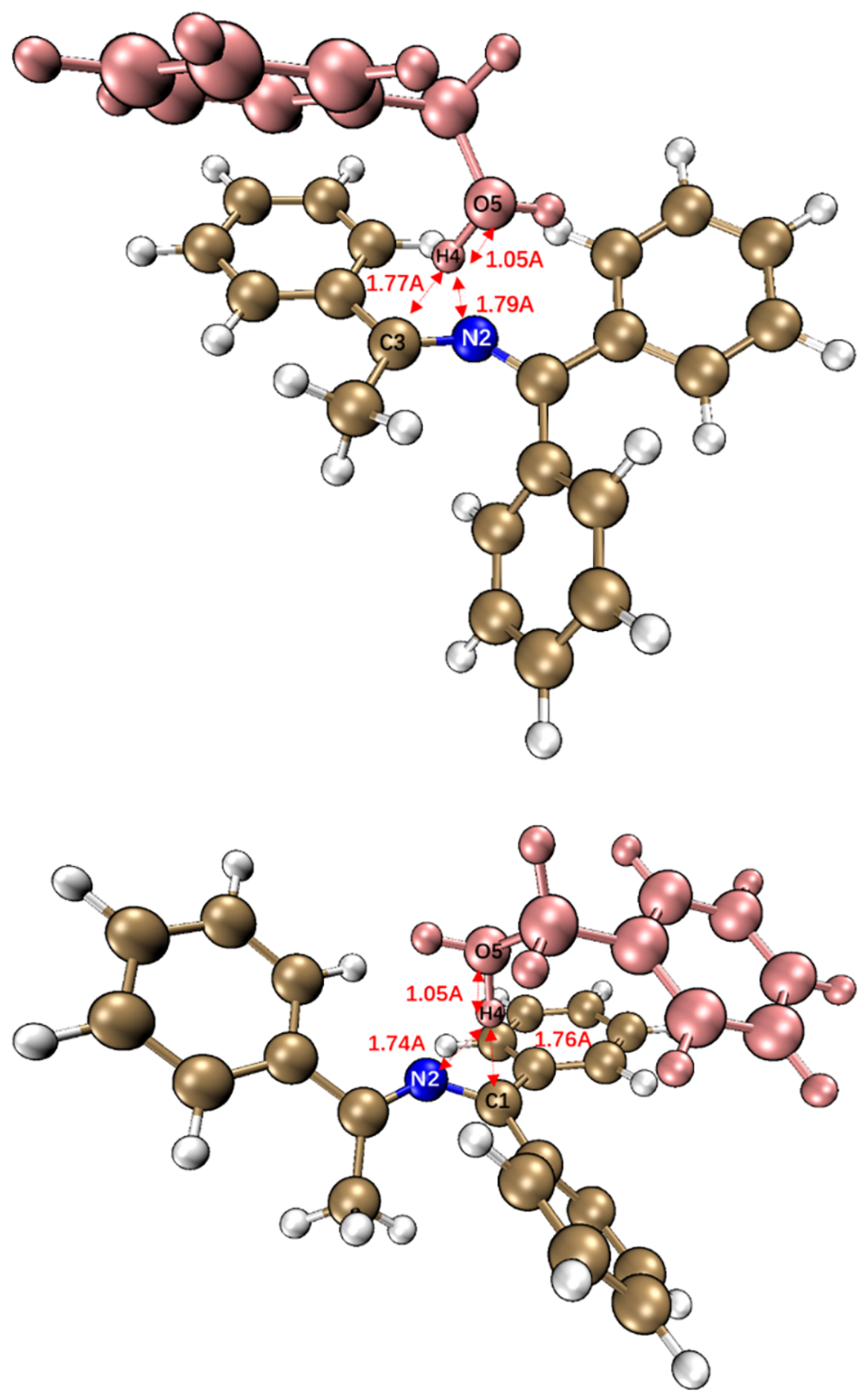

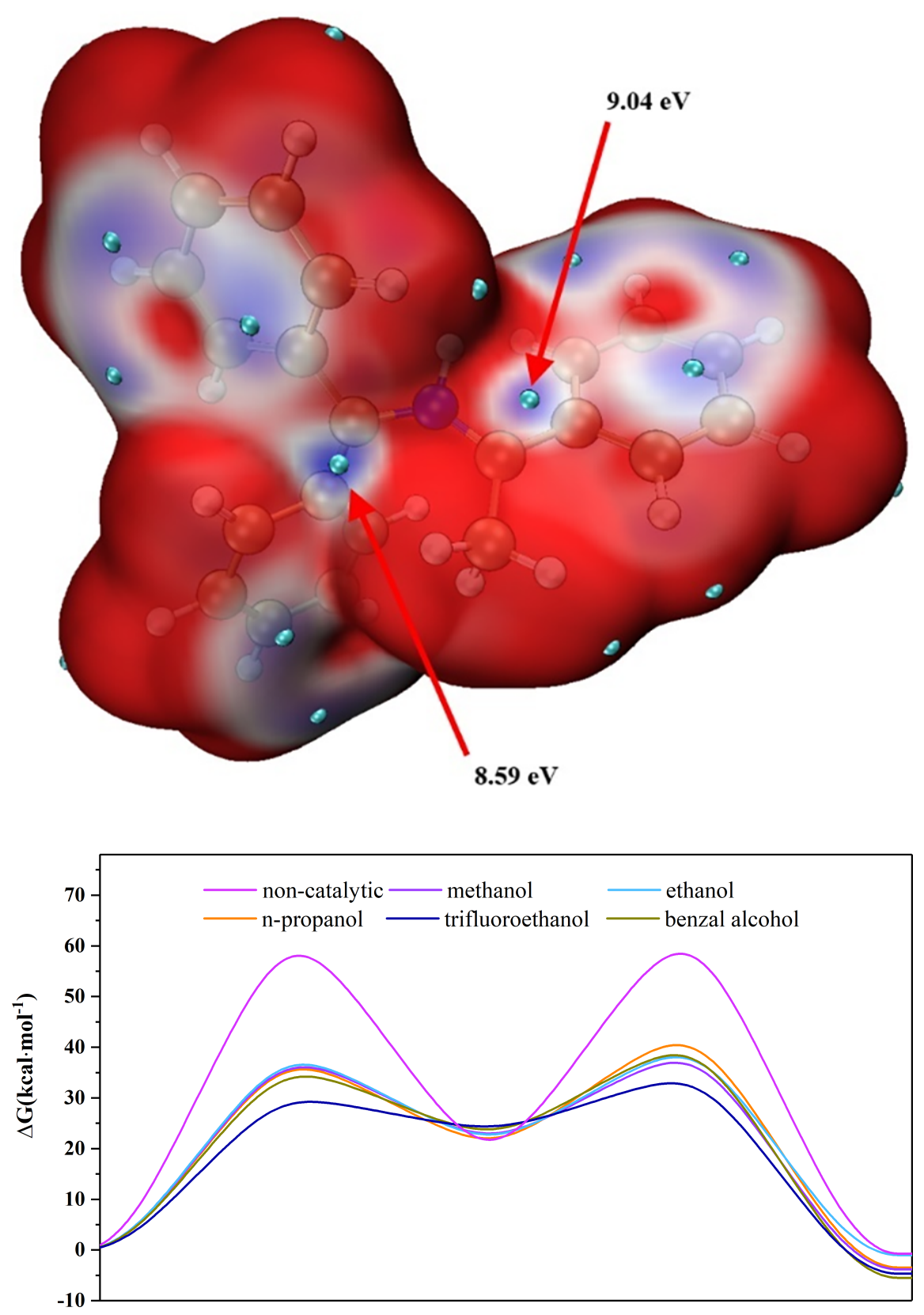